\title{
More on a question of M. Newman on isomorphic subgroups of solvable groups
}

\author{
George Glauberman, \\ Department of Mathematics, University of Chicago, \\ 5734 S. University Avenue, \\ Chicago, IL 60637, \\ \& \\ Geoffrey R. Robinson, \\ Institute of Mathematics, \\ University of Aberdeen, \\ Aberdeen AB24 3UE
}

June 30, 2021

\begin{abstract}
We make further remarks on a question of Moshe Newman, which asked whether it is the case that if $H$ and $K$ are isomorphic subgroups of a finite solvable group $G$ and $H$ is maximal in $G$, then $K$ is also maximal. This continues work begun in [1] by I.M. Isaacs and the second author.

We prove here that if Newman's question has a negative answer for the triple $(G, H, K)$ (ie $H$ is maximal in $G$, but the isomorphic subgroup $K$ is not), then $p \leq 3$ and, for $q=5-p$, we have

$$
O_{q^{\prime}}(H)=O_{q^{\prime}}(K)=O_{q^{\prime}}(G)
$$

and Newman's question also has a negative answer for the triple $\left(G^{*}, H^{*}, K^{*}\right)$, where $G^{*}=G / O_{q^{\prime}}(G)$, etc.. Furthermore, we prove that $G$ has a homomorphic image $\bar{G}$ such that Newman's question has a negative answer for the triple $(\bar{G}, \bar{H}, \bar{K})$, while $F(\bar{G}), F(\bar{H})$ and $F(\bar{K})$ are all $q$-groups, and $O_{\{2,3\}}(\bar{H})$ involves $\mathrm{Qd}(q)$.

As an application, we prove that if $G$ is a finite solvable group such that $H$ and $K$ are isomorphic subgroups of $G$ with $H$ maximal and $K$ not maximal, with $[G: H]=[G: K]$ a power of the prime $p$, then $p \leq 3$ and a Hall $\{2,3\}$-subgroup $L$ of $H$ necessarily involves $S_{3}, A_{4}$ and a nonAbelian group of order 8 (in fact, $L$ involves at least one of $S_{4}$ or $\mathrm{Qd}(3)$ ). In particular, $L$ is neither 2 -closed nor 3 -closed.
\end{abstract}




\section{Introduction}

In this note, we make further remarks on a question of Moshe Newman, which asked whether it is the case that if $H$ and $K$ are isomorphic subgroups of a finite solvable group $G$ and $H$ is maximal in $G$, then $K$ is also maximal. This continues work begun in [1], where it was proved that this is true if $H$ either has Abelian Sylow 2-subgroups or a Sylow tower, and it was noted (in Theorem 3 of that paper) that in a minimal counterexample $G$, we have $O_{p}(G)=1$, where $[G: H]$ is a power of the prime $p$.

\section{Notation, Assumed Background, and Prelim- inary Results}

Recall that a finite group $L$ is said to be involved in the finite group $G$ if there is a subgroup $H$ of $G$ and a normal subgroup $K$ of $G$ such that $H / K \cong L$. We will make frequent use of the first lemma throughout.

Lemma 1: Let $X$ be a finite solvable group, and $\pi$ be a set of primes. Let $T$ be a finite $\pi$-group and let $Y$ be a Hall $\pi$-subgroup of $X$. Then $T$ is involved in $X$ if and only if $T$ is involved in $Y$.

Proof: It is clear that $T$ is involved in $X$ if $T$ is involved in $Y$. We prove the opposite implication by induction on $|X|$. Suppose that $U, V$ are subgroups of $X$ with $V \triangleleft U$ and $U / V \cong T$. Then $T$ is certainly involved in $U$.

If $|U|<|X|$, then $T$ is involved in a Hall $\pi$-subgroup of $U$ by induction. But any Hall $\pi$-subgroup of $U$ is conjugate to a subgroup of $Y$, so that $T$ is involved in $Y$. Hence we may suppose that $U=X$ and that $V \triangleleft X$ with $X / V \cong T$.

Let $M$ be a minimal normal subgroup of $X$ contained in $V$. Then $M$ is either a $\pi$-group or a $\pi^{\prime}$-group. Now $T \cong(U / M) /(V / M)$ so that $T$ is involved in $X / M$. By induction, $T$ is involved in the Hall $\pi$-subgroup $Y M / M$ of $X / M$.

If $M$ is a $\pi$-group then $Y / M$ is a Hall $\pi$-subgroup of $X / M$ and we are done since we already remarked that $T$ is involved in $Y / M$. If $M$ is a $\pi^{\prime}$-group, then $Y M / M \cong Y$, so $T$ is involved in $Y$, and the proof of the lemma is complete.

Let $r$ be a prime. The group $\operatorname{Qd}(r)$ is the semi-direct product of the natural module for $\mathrm{SL}(2, r)$ with $\mathrm{SL}(2, r)$. Note that $|\mathrm{Qd}(r)|=r^{3}\left(r^{2}-1\right)$ and that $\mathrm{Qd}(2)$ is isomorphic to the symmetric group $S_{4}$. Note also that $\mathrm{Qd}(3)$ is a $\{2,3\}$-group. We recall that $\mathrm{Qd}(r)$ is solvable if and only if $r<5$. In this note, the more elementary fact that $|\mathrm{Qd}(r)|$ has at least three prime divisors when $r>3$ usually suffices for our purposes. For $r-1$ and $r+1$ can't both be powers of 2 when $r$ is a prime greater than 3 .

Using Lemma 1, we note that if $\mathrm{Qd}(r)$ is involved in a solvable group $X$, then $r<5$ and $\mathrm{Qd}(r)$ is involved in a Hall $\{2,3\}$-subgroup of $X$. 
Using Theorems of Stellmacher and Glauberman, and Lemma 1, for each finite $r$-group $R$, there is a characteristic subgroup $W(R)$ of $R$ (which is nontrivial whenever $R$ is non-trivial) such that whenever $X$ is a finite solvable group with Sylow $r$-subgroup $R$, then we have $X=O_{r^{\prime}}(X) N_{X}(W(R))$ and furthermore $O_{r^{\prime}}(X) W(R)$ is characteristic in $X$, unless, perhaps, $r<5$ and $\mathrm{Qd}(r)$ is involved in a Hall $\{2,3\}$-subgroup of $X$. When $r$ is odd, we may take $W(R)=Z J(R)$.

When $n$ is an integer, we let $\pi(n)$ denote the set of prime divisors of $n$. We will make frequent use of the following easy lemma:

Lemma 2: Let $X$ be a finite solvable group and $\pi$ be a set of primes. Let $Y$ be a subgroup of $X$ with $\pi([X: Y]) \subseteq \pi$. Then $O_{\pi}(Y) \leq O_{\pi}(X)$.

Proof: Let $U$ be a Hall $\pi$-subgroup of $X$ containing $O_{\pi}(Y)$. Since $Y$ contains a Hall $\pi^{\prime}$-subgroup of $X$, we have $X=Y U=U Y$. Now we have

$$
O_{\pi}(X)=\cap_{x \in X} U^{x}=\cap_{y \in Y} U^{y} \geq O_{\pi}(Y),
$$

as claimed.

Corollary 3: Let $X$ be a finite solvable group and $Y$ be a subgroup of $X$ whose index is a power of the prime s. Let $r$ be a prime divisor of $X$ different from $s$. Then $O_{\{r, s\}}(Y)$ and $O_{\{r, s\}}(X)$ have a common Sylow r-subgroup.

Proof: By Lemma 2, applied with $\pi=\{r, s\}$, we have $O_{\{r, s\}}(Y) \leq O_{\{r, s\}}(X)$. Note that $Y$ contains a Sylow $r$-subgroup of $X$, so that $Y$ certainly contains a Sylow $r$-subgroup $R$ of $O_{\{r, s\}}(X)$. Now

$$
R \cap Y \leq O_{\{r, s\}}(X) \cap Y \leq O_{\{r, s\}}(Y),
$$

so that $O_{\{r, s\}}(Y)$ contains a Sylow $r$-subgroup of $O_{\{r, s\}}(X)$. On the other hand, since $O_{\{r, s\}}(Y) \leq O_{\{r, s\}}(X)$, a Sylow $r$-subgroup of $O_{\{r, s\}}(Y)$ is contained in some Sylow $r$-subgroup of $O_{\{r, s\}}(X)$.

\section{$3 \quad$ Statement and Proof of Theorem A}

Theorem A: Let $H$ be a maximal subgroup of the finite solvable group $G$ and suppose that $[G: H]=p^{a}$ where $p$ is a prime and $a$ is a positive integer. Let $K$ be a subgroup of $G$ which is isomorphic to $H$. Suppose that $K$ is not maximal in $G$.

Then $p \leq 3$, and, for $q=5-p$, we have

$$
O_{q^{\prime}}(H)=O_{q^{\prime}}(G)=O_{q^{\prime}}(K)
$$

and, for $G^{*}=G / O_{q^{\prime}}(G)$, etc., $H^{*}$ and $K^{*}$ are isomorphic subgroups of $G^{*}$ with $H^{*}$ maximal and $K^{*}$ not maximal.

Proof: Let $\phi: H \rightarrow K$ be an isomorphism. 
We proceed by induction on $|G|$. Note that if $N$ is a $\phi$-invariant normal subgroup of $H$ then

$$
N=N \phi \triangleleft H \phi=K
$$

and then $N \triangleleft\langle H, K\rangle=G$. Then $\phi$ induces an isomorphism between $H / N$ and $K / N$, and $H / N$ is maximal in $G / N$, but $K / N$ is not maximal in $G / N$.

Using Theorem 3 of [1] and Lemma 2, we may conclude that $O_{p}(G)=1$. For if not, then we have $O_{p}(G) \subseteq O_{p}(G) \cap H$ by the former result, and both $O_{p}(H) \leq O_{p}(G)$ and $O_{p}(K) \leq O_{p}(G)$ by the latter. Then we have

$$
O_{p}(G)=O_{p}(H)
$$

and

$$
O_{p}(H) \phi=O_{p}(K) \leq O_{p}(G)
$$

Since

$$
\left|O_{p}(G)\right|=\left|O_{p}(H)\right|=\left|O_{p}(K)\right|
$$

we have

$$
O_{p}(G) \phi=O_{p}(G)
$$

so we may apply the argument above with $O_{p}(G)$ in the role of $N$.

We may suppose by induction that the theorem holds for the triple $(G / N, H / N, K / N)$. Then $p \leq 3$ since $[G / N: H / N]=[G: H]$, and we note that

$$
G / O_{q^{\prime}}(G) \cong(G / N) / O_{q^{\prime}}(G / N)
$$

for $q=5-p$. Hence the theorem holds for $G$ in this case. Thus we may suppose that $O_{p}(G)=1$.

Since $O_{p}(G)=1$ and (by Lemma 2) $O_{p}(H) \leq O_{p}(G)$, we see that $F(H)$ is a $p^{\prime}$-group, as is the isomorphic group $F(K)$. Also, there is a prime $r \neq p$ such that $O_{r}(H) \neq 1$.

Suppose first that $r>3$. Then by Lemma 1, we have

$$
O_{\{r, p\}}(H) \leq O_{\{r, p\}}(G)
$$

and likewise,

$$
O_{\{r, p\}}(K) \leq O_{\{r, p\}}(G) .
$$

Furthermore, by Corollary 3 we may suppose (possibly after replacing $K$ by a conjugate) that $O_{\{r, p\}}(H), O_{\{r, p\}}(G)$ and $O_{\{r, p\}}(K)$ all have a common Sylow $r$-subgroup, say $S$.

Since $O_{p}(H)=O_{p}(K)=O_{p}(G)=1$, we see that $W(S)$ is characteristic in each of $O_{\{r, p\}}(H), O_{\{r, p\}}(K)$ and $O_{\{r, p\}}(G)$, where $W(S)$ is the GlaubermanStellmacher characteristic subgroup of $S$ (for note that $O_{\{r, p\}}(G)$ does not involve $\mathrm{Qd}(2)$ or $\mathrm{Qd}(3)$ since $r>3$, and likewise for $O_{\{r, p\}}(H)$ and $\left.O_{\{r, p\}}(K)\right)$. Thus $W(S) \triangleleft G$. Furthermore,

$$
O_{\{r, p\}}(H) \phi=O_{\{r, p\}}(K)
$$


and composing $\phi$ with an inner automorphism of $K$ if necessary, we may suppose that $S \phi=S$, in which case $W(S) \phi=W(S)$. Then $\phi$ induces an isomorphism between $H / W(S)$ and $K / W(S)$.

By induction, the theorem holds for $G / W(S)$. In particular, $p \leq 3$, and, setting $q=5-p$, we see that $W(S)$ is a $q^{\prime}$-group, and that the Theorem therefore holds for $G$.

Hence we may suppose that $F(H)$ is a $q$-group for some prime $q \leq 3$. If $p \neq 5-q$, we may argue as above that $W(T)$ is normal in each of $O_{\{q, p\}}(H), O_{\{q, p\}}(G)$ and $O_{\{q, p\}}(K)$, where $T$ is a common Sylow $q$-subgroup of $O_{\{q, p\}}(H), O_{\{q, p\}}(G)$ and $O_{\{q, p\}}(K)$. This time, $O_{\{q, p\}}(G)$ does not involve $\mathrm{Qd}(2)$ or $\mathrm{Qd}(3)$ since $p>3$. But that leads to a contradiction, since the theorem holds for $G / W(T)$ and then we see that $[G: H]=[G / W(T): H / W(T)]$ is either a power of 2 or power of 3 , so $p \leq 3$, contrary to current assumptions.

Now we may suppose that $p \leq 3$ and that $F(H)$ is a $q$-group, where $q=5-p$. Since $[G: H]$ is a power of $p$ and $O_{p}(G)=1$, we have $F(G) \leq H$, so that $F(G) \leq F(H)$ and $F(G)$ is a $q$-group. Since $H \cong K$, we also see that $F(K)$ is a $q$-group. The proof of Theorem $\mathrm{A}$ is complete.

\section{Statement and Proof of Theorem B and some consequences}

Theorem B: Let $H$ be a maximal subgroup of the finite solvable group $G$ and suppose that $[G: H]=p^{a}$ where $p \leq 3$ is a prime and $a$ is a positive integer. Let $K$ be a subgroup of $G$ which is isomorphic to $H$. Suppose that $K$ is not maximal in $G$ and that $F(H), F(K)$ and $F(G)$ are all $q$-groups, where $q=5-p$. Let $Q$ be a Sylow q-subgroup of $H$.

Then $G$ has a homomorphic image $G^{*}$ such that $H^{*}$ and $K^{*}$ (the respective images of $H$ and $K)$ are isomorphic subgroups of $G^{*}$ with $H^{*}$ maximal and $K^{*}$ not maximal, and with $F\left(H^{*}\right), F\left(K^{*}\right)$ and $F\left(G^{*}\right)$ all q-groups. Furthermore, $O_{\{2,3\}}\left(H^{*}\right)$ involves $\mathrm{Qd}(q)$ and no non-identity characteristic subgroup of $Q^{*}$ is normal in $H^{*}$.

Proof: Let $\phi: H \rightarrow K$ be the isomorphism as before. We may, and do, suppose that $Q \phi=Q$, composing $\phi$ with an inner automorphism of $K$ if necessary.

Using Theorem A, we may suppose that there is no non-trivial $\phi$-invariant normal subgroup of $H$. For otherwise, if $1 \neq N \triangleleft H$ is $\phi$-invariant and chosen of maximal order subject to these conditions, then $N=N \phi \triangleleft H \phi=K$, so $N \triangleleft\langle H, K\rangle=G$ and $\phi$ induces an isomorphism between $H / N$ and $K / N$. By Theorem A, we have

$$
O_{q^{\prime}}(H / N)=O_{q^{\prime}}(G / N)=O_{q^{\prime}}(K / N)=1
$$


by the maximal choice of $N$ (hence $F(H / N), F(K / N)$ and $F(G / N)$ are all $q$ groups) and the theorem holds for $G / N$ by induction, so it holds for $G$.

Suppose now that $O_{\{2,3\}}(H)$ does not involve $\mathrm{Qd}(q)$. Then as before, $Q$ contains a common Sylow $q$-subgroup $T$ of $O_{\{2,3\}}(H), O_{\{2,3\}}(K)$ and $O_{\{2,3\}}(G)$ such that $W(T) \triangleleft H$ and $W(T) \phi=W(T)$, contradicting the fact that there is no non-trivial $\phi$-invariant normal subgroup of $H$. Hence $O_{\{2,3\}}(H)$ involves $\operatorname{Qd}(q)$.

If there is a non-identity characteristic subgroup $S$ of $Q$ which is normal in $H$, then we have $S=S \phi$ since $Q=Q \phi$, again contradicting the fact that no non-trivial $\phi$-invariant normal subgroup of $H$. The proof of Theorem B is complete.

We may combine Theorems A and B to deduce:

Corollary C: Let $G$ be a finite solvable group and $H, K$ be isomorphic subgroups of $G$ such that $H$ is maximal but $K$ is not, and with $[G: H]=p^{a}$ for some prime $p$ and positive integer $a$. Suppose (with no loss of generality) that $H \cap K$ contains a Hall $p^{\prime}$-subgroup of $G$ (this is just a matter of replacing $K$ by a conjugate if necessary). Then $p \leq 3$. Let $\phi: H \rightarrow K$ be an isomorphism, chosen so that $Q \phi=Q$ for a Sylow $q$-subgroup $Q$ of $H$, where $q=5-p$ (this can be achieved by composing $\phi$ with an inner automorphism of $K$ if necessary). Let $C$ be the unique maximal $\phi$-invariant normal subgroup of $G$ which is contained in $H \cap K$, and let $G^{*}=G / C$, etc.. Then $F\left(H^{*}\right), F\left(K^{*}\right)$ and $F\left(G^{*}\right)$ are all q-groups. Also, $\operatorname{Qd}(q)$ is involved in $O_{\{2,3\}}\left(H^{*}\right)$, and no non-identity characteristic subgroup of $Q^{*}$ is normal in $H^{*}$.

Proof: The proofs of Theorems A and B show that unless $G$ itself can play the role of $G^{*}$, there is a non-identity $\phi$-invariant normal subgroup $N$ of $G$ contained in both $H$ and $K$ such that $H / N$ and $K / N$ are isomorphic with $H / N$ maximal in $G / N$ and $K / N$ not maximal. In that case, since $N \leq C$, we may suppose that the Theorem holds in $G / N$ by induction, and then it holds in $G$.

Corollary D: Let $G$ be a finite solvable group containing isomorphic subgroups $H$ and $K$ such that $H$ is maximal in $G$ but $K$ is not. Then $[G: H]=[G: K]$ is a power of a prime $p \leq 3$ and a Hall $\{2,3\}$-subgroup $L$ of $H$ involves $\mathrm{Qd}(q)$, where $q=5-p$. In particular, $L$ involves $S_{3}, A_{4}$ and a non-Abelian group of order 8 , so that $L$ is neither 2 -closed nor 2-nilpotent.

\section{References}

[1] Isaacs, I.M. \& Robinson, G.R., Isomorphic subgroups of solvable groups, Proc Amer. Math. Soc 143 (2015) 3371-3376. 